\section{The Neppe-Close triadic dimensional vortical paradigm: An invited summary}

\author{
Vernon M Neppe* and Edward R Close
}

Pacific Neuropsychiatric Institute, Seattle, WA, USA

\section{Abstract}

Physicists are generally trained in the Standard Model of Physics (SMP). This implies that they perceive and account for only 3 dimensions of space in a moment in time (3S-1t) (a 4-dimensional [4D] model). However, applying the SMP, more than fifty significant conundrums have arisen that are unexplained or incomplete. Explaining these within the SMP 4D fabric led to hypothesizing a 'fifth force', most recently the hypothetical 'X17 particle'. We propose this hypothetical X17 may better be explained by a 9-dimensional model (9D) with gimmel. Our model, the Neppe-Close Triadic Dimensional Vortical Paradigm (TDVP) has amplified the 'physics' from 4 dimensions to 9D, specifically first postulating and then further demonstrating mathematicallystarting with derivations of the Cabibbo angle-that 9 dimensions must exist. Moreover, this data is empirically demonstrated because the neutron, proton and electron mass-energy-gimmel equivalence in the Triadic Rotational Units of Equivalence (TRUE) as part of the TDVP model, exactly corresponds with the normalized data for the mass-energy equivalence volumetric data for these particles in the CERN Large Hadron Collider. This data shows definitively that we exist in a 9-dimensional finite, quantized, volumetric, spinning reality. This is, furthermore, embedded in an infinite continuity $(9 D+)$. Mathematically, applying this $9 D+$ model definitively requires an extra third component that is massless and energyless ('gimmel'). Without gimmel, no particle in the universe would be stable. TDVP unifies nature because the same laws apply across the quantum, macro-world and cosmological reality. Our 4D experience is simply the physical component of $9 \mathrm{D}+$ existence.

Summary Amplification: At all levels, there is the consistent application of a 9-Dimensional quantized finite reality embedded within an infinite continuity. The application of gimmel specifically requires applying the 9-dimensional model and is based on necessary mathematical calculations not only at the quantal level (where the fifty plus unsolved, unexplained or contradictory conundrums can be explained somewhat, and there is no longer 'quantum weirdness'), but at the macroscale level with more gimmel in the life elements (which, additionally, are consistently all cubic multiples of 108 cubed), as well as cosmologically, where the correlations with proportionate Dark Matter and Dark Energy are overwhelming. Moreover, these 9-dimensional plus factors together with Triadic Rotational Units of Equivalence (TRUE) and gimmel, allow numerous solutions that couldn't otherwise be solved. For example, importantly, applying the simple mathematics of TRUE, we can demonstrate why gluons, while adequate in $4 D$, are impossible applying 9D. These solutions are simpler because we have markedly adapted George SpencerBrown's 'Laws of Form' to applying a new method of mathematical calculation, Edward Close's 'Calculus of Distinctions' (COD) which recognizes quantal limits and that the nature of finite reality is quantized and volumetric. The COD includes distinguishing between content, extent, and impact. We emphasize the pioneering works of Wolfgang Pauli with his multidimensional model and his 'Pauli Exclusion Principle', Alfred Whitehead with 'Process Philosophy' and his 'Principia Mathematica' (with Bertrand Russell), Georg Cantor with Set Theory, and Roger Penrose with spinors and twistors. TDVP is a prime example of our broad new specialty of 'Dimensional Biopsychophysics' (DBP). DBP extends physics, consciousness, and the biopsychosocial to extra dimensions and applies mathematics empirically. Like Max Tegmark, we recognize the key role of mathematics as fundamental in nature, not just for application in calculation and operations.

\section{More Information}

*Address for Correspondence: Vernon $M$ Neppe MD, PhD, FRSSAf, DFAPA, Pacific Neuropsychiatric Institute, $4616,25^{\text {th }}$ Ave NE, \#236 Seattle, WA 98105, USA, Tel: 206527 6289; 206527 6289; Email: Psyche@pni.org

Submitted: 07 December 2019

Approved: 10 January 2020

Published: 13 January 2020

How to cite this article: Neppe VM, Close ER. The Neppe-Close triadic dimensional vortical paradigm: An invited summary. Int J Phys Res Appl. 2020; 3: 001-014.

DOI: dx.doi.org/10.29328/journal.ijpra. 1001018

ORCiD: orcid.org/0000-0001-8621-8796

Copyright: (c) 2020 Neppe VM, et al. This is an open access article distributed under the Creative Commons Attribution License, which permits unrestricted use, distribution, and reproduction in any medium, provided the original work is properly cited.

Keywords and terms: 3S-t; 9D; 9D+; 4D; Calculus of distinctions; Calculus of dimensional distinctions; Cabibbo; Cantor; Close; consciousness; Consciousness research; Continuous; Dark matter; Dark energy; Definition; Dimensional Biopsychophysics; Dimensions; Discrete; Distinctions; Diophantine equations; Entanglement; Energy; Erroneous; Framework model; Framework of gimmel; Immediacy; Infinite continuity; Infinity; Information; Krasznahorkay; Limitations; Mass; Mathematics; Misinterpretation; Neppe; Ordropy; Organic; Pauli; Pauli exclusion principle; Penrose; Periodic table; Phenomenological levels; Physics; Quantum; Quantum relative to; Relative; Relative nonlocality; Restricted 3S-1t; Russell; Set theory; Shells; Space; Spencer brown; transfinite; Triadic dimensional distinction vortical paradigm; Spinors; TDVP; Tegmark; Triadic Dimensional Distinction Vortical Paradigm; Triadic Rotational Units of Equivalence (TRUE); Twistors; Valence; Volumetric; Vortices; X17 particle; Whitehead

W) Check for updates

OPEN ACCESS

\section{Introduction}

Most physicists abide by the Standard Model of Physics: They are taught to perceive the quantal universe as separate in laws to the macro-universe. Moreover, these governing laws are accepted as different from the rules relating to the cosmological universe. Effectively, it might be that quantal mechanics, our regular world and the cosmological realities almost by definition seem to be governed by their own independent laws. These scientists might, nevertheless, recognize contradictions, conundrums and unexplained concepts, and even understand that these laws might represent 
limited pieces of an incomplete jigsaw puzzle. Nevertheless, they might think there is nothing they can do: "It's just how it is. It shows that nature is not inherently and consistently logical and we must simply accept that fact."

In this paper, we're trying to bridge a gap between 4 and 9 dimensions, with additionally, the 'infinite continuity' combined with the 'discrete finite'.

With great respect, the authors have recognized that the laws of nature require significant additions to be unified and internally consistent. This means the information in this paper moving from 4D to 9D physics does not just require minor changes. Certain contradictions exist and many phenomena are unexplained, and although the ideas we discuss below are based on math and empirical science, the usual physicist trained only in a 4-dimensional model of experiencing reality, might see the 9-dimensional work we've pioneered as "speculative from our physical point of view". That same physical view to us appears truncated or filtered, and, we argue might be cogently explained if one examines a broader reality.

We're trying to clarify how scientists who've been trained in the current paradigm of the Standard Model of Physics (SMP), might see their paradigm as almost perfect and just needing to be fine-tuned. However, we see the SMP as markedly imperfect and needing to be extended and expanded across dimensions. We have called this broader discipline 'Dimensional Biopsychophysics'. Therefore, we have needed to apply some terminology that is new and possibly unfamiliar to the reader. However, we recognize that we must make our laws of nature work together as one. We think we have done this.

\section{Historical background}

For more than a century, scientists have attempted without success to develop a 'theory of everything' [1-4]. For some physicists this has been restricted solely to finding solutions in quantum physics. However, others have ignored extending this to dark matter and dark energy, and some have concentrated purely on the cosmological not the quantal mechanical. Biologists have recognized life and often ignored the quantal and cosmological. Still other researchers have focused mainly on the elements that are involved in terms of inorganic and organic chemistry. Then there are those who have purely studied consciousness without evaluating these other areas. These have all reflected mysteries that have befuddled even Einstein [5], who supposedly spent much of the last 20 years of his life [6-9], trying to find a theory that integrated all known forces [10].

This attempt at creating a model that explains information of various facets of physics has been referred to as the Unified Field Theory (UFT) [11]. Physicists have hoped to construct this UFT theory [11], which would coherently explain quarks and subatomic particles through to all cosmic forces including the formation of galaxies and dark matter and energy and so unify all of finite reality [11-13].

The much-desired Unified Field Theory [11], concept is sometimes referred to as a "Theory of Everything" (TOE) [14], A TOE is a commonly applied term, but ambiguous in regard to more than one context (physical or general) for a complete explanatory model of reality conforming to the laws of nature. TOEs should seamlessly reconcile with all the major theoretical models and authoritative sources of all the sciences and mathematics, but should not be construed as reflecting omniscience, instead implying application of universal principles. TOEs are sometimes regarded as primarily philosophical, yet with the original, limited meaning related exclusively to Physics [15]. We disagree with the term 'TOE' because it is ambiguous, and its use can be misinterpreted. We've instead proposed the term Metaparadigm [16]. This refers to the broadest paradigm impacting all sciences, mathematics and philosophy.

In 2011, the authors proposed such a metaparadigm, and called it the 'Triadic Dimensional Vortical Paradigm' (or TDVP) also synonymously called Triadic Dimensional (Distinction) Vortical Paradigm TDdVP, because it necessarily involves the Dimensional Triads of Space-time-consciousness in rotating movements (vortices) [17].

The predecessors of the early involvement of consciousness, mathematics and physics?

We link up here several earlier thinkers: Alfred North Whitehead, English mathematician and philosopher, [18-20] with Bertrand Russell [21,22], Georg Cantor [23] and George Spencer Brown [24]. These great names lead to some later thinkers like David Chalmers, who recognized psychophysical law' [25,26] and Abner Shimony [27] modifying Whitehead $[18,21,22]$ who confronted the problems of consciousness head on. Additionally, Max Tegmark, [28] like the authors, and following on ancient Greeks like Pythagoras and Plato [29-31] (but with a retrospectoscope of modernity!) has been prepared to argue for mathematics being fundamental to nature and reality and not just a calculus or operation $[15,32$, 33].

Consciousness is recognized more than before even though it's not the prevailing view! [15 p227].

The idea of the quantum frames of reference with consciousness having direct relationships with mathematics and physics is therefore not new. Even in 1929, Whitehead, who is best known as the defining figure of the philosophical school known as 'process philosophy' [18-20] posited that quantum mechanics perceived the universe as a process of events, at least some of which are imbued with a mental quality ("throbs, or occasions of experience") [18,21]. Whitehead's polymathic contributions have today found application to a 
wide variety of disciplines, including not only mathematics, logic, and physics, but ecology, theology, education, physics, biology, economics, and psychology. However, likely Whitehead's most notable work in these fields is the threevolume 1910-1913 Principia Mathematica (PM) [22] (with numerous revisions thereafter), written in modernized logical notation with his former student Bertrand Russell. Whitehead and Russell introduced a complex system now called "the ramified theory of types" [21]. After the introduction of a theory of sets, or 'classes', the system of PM can be compared with the early development of Georg Cantor's Set Theory [23]. Whitehead's work also precedes, though is very different from the authors 'Close's Calculus of Distinctions' (COD) [34-37]. COD applies the most basic methods of logic, and recognizes these sets have empirical bases and that there are limits to the Newtonian-Leibnizian infinitesimal calculus' [38,39] With the COD, much of nature can be handled as integers, simplifying mathematical interpretations considerably [40].

George Spencer Brown in his 1969 classic book Laws of Form [24] amplified several of these mathematicalphilosophical predecessors of the structure of reality and brought a third type that is into logic, that is equivalent to the imaginary (or complex) numbers in pure math. This was the key component that led to a breakthrough by the authors in Close's Calculus of Distinctions [41] and then the Calculus of Dimensional Distinctions [37]. This CoDD allows for a workable mathematicologic model across dimensions and consciousness and its empirical base involves inter alia, normalization of the electron to 1 when applying Quantum Triadic Rotational Units of Equivalence (TRUE) [42-44]. This is now fundamental to many of the empirical proofs of the Triadic Dimensional Vortical Paradigm. Therefore, much has happened since the initial philosophical mathematical concepts of Cantor [45,46] and of Whitehead and Russell [22].

\section{The triadic dimensional vortical paradigm}

TDVP is now sometimes referred to as the most complete of the "Theories of Everything" (TOEs) [14], because, after thorough detailed comparative analysis, it fits a broader range of objective criteria.

For example, when applying objective, peer- reviewed metric comparisons to 24 TOEs, TDVP scores far the highest with a perfect score (39/39) [16]. However, these listed criteria are relatively non-specific and notably do not include the unifying of gravitation and relativity. Extending TDVP in later work, applying even broader criteria, Triadic Dimensional Vortical Paradigm still scores 'perfectly' even with some seventy broad criteria $[47,48]$. There remain unexplained areas in this model, but the TOE of TDVP is far, far closer than scientific exploration has ever been.

With respect, we have scientific evidence that our 'gimmel' discovered through mathematical analysis, and our '9-dimensional' scientific proofs explain many unsolved conundrums, and moreover, unify the quantal with the macroworld and cosmology $[47,48]$.

\section{We explain these briefly:}

'Dimensional Biopsychophysics' (DBP) is the term we developed in 2014 [15] for the broad new specialty recognizing the need for extra dimensions, and incorporating 'consciousness' in its broadest context, including consciousness outside the brain. DBP extends physics, consciousness, and the biopsychosocial, and applies mathematics empirically. The TDVP model is a prime example of DBP. Initially, in 2011, we did not know for certain how many dimensions were involved but we postulated that there had to be specifically 9 finite quantized dimensions. We then proved that hypothesis mathematically. Moreover, we also realized that for our model to be complete, there had to be something different outside the finite dimensional box. That required postulating an infinite continuity that was part of the whole, and it fitted with Georg Cantor's ideas of infinity and the infinity of infinities [23] as well as maintaining a way to provide a consistent logical theory that would not compromise Gödel's Incompleteness Theorem (GIT) [49].

9-dimensional finite reality reflects our existence as measured by the extent in Space, Time and a third dimensional substrate which we propose is 'Gimmel-Consciousness'. These are all tethered together geometrically so that the concept of 'Minkowski Space-Time' [49] has now been extended to [50]. 'Space-Time-Gimmel Consciousness' (STC) [47,48]. STC incorporates our physical experience of 3S-1t (3 spatial dimensions in a single quantum of time, namely the present ' $1 \mathrm{t}$ '). However, $3 \mathrm{~S}-1 \mathrm{t}$ is recognized as embedded within the 9-dimensional finite reality $[47,48]$. While we have not yet defined exactly what these 9 dimensions are, that is not a critical aspect of TDVP: However, based on the supporting math and logic, we have proposed that the three Spatial dimensions extend far beyond the physical. More controversially, we propose that there are 3 dimensions of Time (not just linear 'past-present-future') and even more so, 3 dimensions of Gimmel-Consciousness [47,48]. These dimensions are dynamic in that they might fluctuate depending on relative circumstances $[47,48,51]$.

'Gimmel' is the newly discovered third substance. We published the mathematical derivation of gimmel in 2015 [51] - gimmel is necessarily massless and energyless,. We use the term Gimmel because we don't know if we're necessarily referring to consciousness itself, or simply a vehicle of consciousness, or some kind of carrier of consciousness. No one has yet offered a viable alternative explanation that does not implicate gimmel with some kind of Consciousness [52].

Whether using the term 'gimmel' or 'consciousness', these concepts are massless, and energyless. They still necessarily and always contribute to the 9D fabrics of atomic structure and substructures at the quantized finite level. 
Mathematically, gimmel necessarily has to exist in union with any particle in the universe for that particle to be stable. Without gimmel, the spinning (vortical) atoms would be unstable and asymmetrical about their axes and would, in effect, fly apart: Our world and the physical universe could not exist [53]. Gimmel is necessarily in union with all stable particles because that allows rotation along axes providing the obligatory atomic stability [52]. Gimmel is proven mathematically and necessary because everything in reality must balance with volumetric calculations and that would not happen if we just had, for example, protons plus neutrons plus electrons equaling atoms. This would create an inequality as the volumetric nature of reality restricts mathematical solutions to the form of specific third order Diophantine Equations [53], relevant in physics to the Close Conveyance Equąion [44,54]. The Close Conveyance equation $\left(\mathrm{X}_{1}\right)^{3}+$ $\left(\mathrm{X}_{2}\right)+\left(\mathrm{X}_{3}\right)^{3}=\mathrm{Z}^{3}$ for triplets is derived by combining quantum particles:

$$
\sum^{\mathrm{n}} \mathrm{i}=1\left(\mathrm{X}_{\mathrm{n}}\right)^{\mathrm{m}}=\mathrm{Z}^{\mathrm{m}} .
$$

This requires balancing of the volumetric integral components $[55,56]$. In the Periodic Table of the Elements, for example, there are always the same specific small number of electrons as protons leaving an equation inequality $2\left(\mathrm{X}_{1}\right)^{3}$ $+\left(\mathrm{X}_{3}\right)^{3}=\mathrm{Z}^{3}$, which calculation can also be tested empirically $[47,48,52]$.

Gimmel and the related 'Triadic Rotational Units of Equivalence' (TRUE) [52] are derived by applying 'normalization' to the measurement parameters of electrons, and up-quarks and down-quarks (the only stable quarks) in the protons and neutrons $[44,57,58]$. In order for the equation to balance, there is the necessity of that massless, energyless "something" (gimmel) to exist in the finite quantized reality, Gimmel allows a necessary and essential solution to the physics equations pertaining to the combination of the elementary vortical particles to form atoms in elements and molecules. Gimmel is proven necessary by applying dimensional analysis across 9-dimensions, and specifically, applying the conventional notion of "natural units" in another step by further including normalizing the electron massenergy to 1 . This model of reality is volumetric, and the Diophantine equations, specifically the Conveyance equation, is the mathematical form required $[47,48,52]$.

Besides the math proofs, these have been empirically demonstrated because the normalized figures for electrons, protons and neutrons in Triadic Rotational Units of Equivalence exactly correspond with the Mass-energy equivalence normalized data in the CERN Large Hadron Collider $[42,59,60]$. Gimmel requires vortical rotations in a 9-dimensional quantized finite reality: This equivalence of measurement units is mathematically impossible in just our $3 S-1 t$ physical reality alone $[42,52,61]$.

However, could some new findings be an alternative? On
23 November 2019, the popular press excitedly reported research from Physicist Attila Krasznahorkay and colleagues at the Hungarian Academy of Sciences about the "fifth law of physical forces supporting the existence of a hypothetical X17 particle" [62]. This "connects our visible world with the dark matter". Jonathan Feng, a professor of physics and astronomy at the University of California at Irvine, pointed out that "if it were true, it would be a Nobel no-brainer" [63].

However, with respect, we propose that the idea of a fifth force after electromagnetism, the strong and weak forces, and gravitation might turn out to be unnecessary. We argue that these researchers might have detected the effects of gimmel $[55,56,64]$ and might find application of the 9-dimensional (9D) matrix $[48,65,66]$. This is important, particularly in the context of the different atomic shells and valences in the Periodic Table $[43,57]$. This possibly impacts the volumetric measurement of the two different angles in two elements that they have described. What is the data on several other elements? What pattern, if any, can be found?

These Krasznahorkay, et al., findings relate to their new discovery of 'X17' and this is regarded as reflecting a new 'force' relating to the Krasznahorkay, et al., research proof [62], based on particles coming off beryllium-8 at around a 140-degree angle. This was 'strange and new'. Their previous work was with Helium where a 115-degree angle was also unexplained. "They're leading us closer to what's considered the Holy Grail in physics, which Albert Einstein had pursued but never achieved" [62]. That quotation is true: Einstein spent the last two decades of his life trying to find in effect extra dimensions but ignored the volumetric nature of rotating elementary particles (just as Planck had done, as well), 9-dimensions specifically, and gimmel [48,65-69]. But the "they're" may refer to others.

This is so because the proven, though not well-known, features of 9D and gimmel have simply not been considered, yet at least could provide a legitimate alternative hypothesis to explain these Hungarian findings better than a new unexplained 'fifth force', that might imply even a sixth or seventh force or more according to Dr. Feng [63]. Moreover, 9D [70] and gimmel $[43,51,56]$ have profound empirical and math explanatory support.

We illustrate this point with a critically important aside: We mathematically demonstrated by calculation why the Cabibbo Mixing angle was $13.04 \pm 0.05$ degrees. This was the first major finding initially demonstrating the necessity of a 9-dimensional quantized finite model. That proof was only demonstrable through a 9-dimensional mathematical derivation, providing the reason why no-one before that time (2014) had been able to do that calculation because they had worked only with 3 S-1t $[14,71]$ or possibly with unsubstantiated theories of multidimensionality like strings and superstrings, which remain unproven and are likely 
fundamentally flawed, because despite at least allowing some ten thousand scientists to seriously contemplate such multidimensional realities over many years, no one has been able to prove any of the models [72-76].

We definitively proved mathematically that 9-dimensional spin model through that careful derivation of the Cabibbo Mixing angle [14]. We applied well-defined physics, wellsubstantiated empirical data, including well defined constants such as the Bohr radius (radius of the hydrogen atom), speed of light, Planck's constant, rest mass of the electron, its radius and charge, the Coulomb constant and $\pi$. With these, we added well-defined equations and principles, such as the Lorentz correction, the principle of conservation of angular momentum, kinetic energy equation, De Broglie's wave equation, Coulomb's equation, the centrifugal force equation, the wave length of a rotating body and calculations of magnetic moment [14]. We applied these to electron rotation and its inherent spin utilizing the basic concepts of a unified space-time-consciousness theory of finite reality from the Neppe-Close Triadic Dimensional Distinction Vortical Paradigm (TDVP or TDdVP as 'distinction' is optional). These included applying two new mathematical techniques that we have developed as part of this TDVP model [14], namely 'dimensional extrapolation' across rotating dimensions [77] and the principles of the 'calculus of distinctions' [37].

Distinctions are very basic ways of conceptualizing separations into different groups. Dr. Close's 'calculus of distinctions' is more than just a non-Newtonian calculus, it subsumes mathematics, set theory and logic under a common umbrella, and integrates these empirically with physics and nature in a way that is unique. The CoD creates a remarkable bridge between elementary symbolic logic and higher level mathematical structures [42,61].

We produced a detailed mathematical derivation of the mixing angle of elementary particle fermions, exemplified by the Cabibbo angle in quarks with the empirical calculation $13.032^{\circ}$. We further showed that this result could only be derived from a 9-dimensional mathematical spin model. This finding also supported a component of the broader Triadic Dimensional Distinction Vortical Paradigm (TDVP) hypothesis [15], namely that the finite reality consists of a 9-dimensional vortical (spinning) geometric ('dimensionometric') structure $[14,71,78,79]$.

Wearguethatresearchersin Dimensional Biopsychophysics might still use 9D and gimmel, but would need to apply a very different method to explain why the angles in Be and He are very different. In this instance, as described, the Hungarian researchers appear to be dealing with physical angles and not just vector space. Nevertheless, it just might be possible that these Krasznahorkay, et al., angles may be calculated applying similar 9-D mathematics using the fundamental TDVP (Triadic Dimensional Vortical Paradigm) principles, although their derivations appear to be disparate compared with the Cabibbo angle derivation $[4,56]$. The key might be finding a consistency in techniques between calculating the 9-dimensional finding of Helium at $115^{\circ}$ and Beryllium at $140^{\circ}$. One approach could be possibly through re-examining the Periodic Table of the Elements in the context of valence and electron shells $[43,57]$ applying Triadic Rotational Units of Equivalence [52].

Let's review the pertinent history, as we can back up our points by the empirical information TDVP explains with mathematical proofs: The first limitation leading to a lack of solutions of previous mathematicians is, in our opinion, a very basic one. The earlier multidimensional researchers (such as Kaluza and Klein [81-85], and Pauli [85] and (and relatively later) Rauscher [86], String theory and Superstring theorists $[73,74,77]$, should have been dealing with volumes as opposed to non-geometrical components and singularities..

We single out the brilliant Nobel physicist Wolfgang Ernst Pauli, the Austrian-Swiss-American theoretical Nobel physicist who pioneered the 'Pauli exclusion principle' [87]. This involved spin theory and was the basis of a theory of the structure of matter. Spin and vortical rotations and electron shells are all pertinent to TDVP and indirectly linked with the Exclusion Principle [88-90]. However, Pauli also worked on developing five- and six-dimensional models until 1953, but didn't publish his findings because he was bothered by the appearance of what he called "...rather unphysical shadow particles [5]”. Pauli's multidimensional work has been largely ignored in comparison with his other great contributions. Unfortunately, Pauli never officially published all this, though he talked about it: it came up, apocryphally, in letters with Carl Jung, but it's well known that he had pursued including more dimensions and went as far as six. And Pauli recognized the 'scientific and epistemological aspects of the ideas of the unconscious and the changes that needed to occur' [8890]. He described 'nature's ghost particles' while describing neutrinos [88]. But, since Pauli's time, science has discovered that just over $95 \%$ of the substance of reality consists of some sort of what Pauli had called 'shadow stuff', presently called "dark energy" and "dark matter" and, not directly detectable through the physical senses or extensions of them [58].

Traditional physics have sometimes tried to collapse their quantum mechanics back down to the $2^{\text {nd }}$ planar or linear $1^{\text {st }}$ dimension when that is purely theoretical, rather than starting with the only empirical, observational reality that spinning particles are volumetric (i.e., 3-dimensional).

Secondly, the most important deficiency might be that previous researchers did not introduce consciousness into the integrative equations of physics $[59,90]$. This, too, we have proven in the 9D model $[59,90]$.

Another important contributor to thinking, consciousness and rotations is the English mathematical theoretical physicist, 
mathematician and philosopher of science, Sir Roger Penrose who described Twistor Algebra in 1967, and spoke of 'spinors' [92]. He has worked with Stuart Hameroff on a complex model incorporating the brain, consciousness, 'mind', and quantum physics $[93,94]$.

Penrose suggested that 'twistor space' should be the basic arena for physics from which space-time itself should emerge. Twistors and spinors $[92,95,96]$ allow powerful mathematical methods of application to differential and integral geometry, nonlinear differential equations and representation theory, and in physics to relativity and quantum field theory, in particular to scattering amplitudes. Mathematically, projective twistor space involves a three-dimensional complex manifold. Twistor theory originally encoded physical fields on 'Minkowski space' $[8,50]$ and then applied twistor space via the 'Penrose transform' of arbitrary spin in massless fields [95,97100]. Twistor string theory was extended first by generalizing the RSV Yang-Mills amplitude formula [101,102] and then by finding the underlying string theory $[74,75]$. There have been other attempts to extend spinors to the "Infinite tension limit of the pure spinor superstring" [103]. We can add Penrose's awareness of the relevance of consciousness [93]. These are all pertinent to the Triadic Dimensional Vortical Paradigm where vortices of rotating movements across 9 dimensions and the infinite continuity and a new easier calculating method of the calculus of distinctions 104 allow extensions of many of these ideas $[34,35,37]$. TDVP describes certainly massless, energyless gimmel, though the extra-dimensional model is beyond typical field theory descriptions $[47,105,106]$ and extends with the infinite continuity enveloping be the empirically demonstrated 9-dimensional finite [15]. We argue that extending spinors and twistors to the empirically feasible Triadic Dimensional Vortical Paradigm and not applying the various String Theories may turn out to be more fruitful and feasible [107-109].

By contrast, the more classical 4D scientists have limited their explanations of the 'quantum probability wave collapse' to 'local' (immediate space-time) effects and ignored the broader extended dimensions and consciousness. In this regard, we have proposed (what we've called) 'Vortical Indivension' (VI) as a downstream (and upstream) dimensional mechanism to explain quantum collapse or superposition [109-111]. Indivension provides the mechanism of the process of communication across, between and within different dimensional domains by interfacing the content vortices, with scalars, vectors and tensors if needed ${ }^{1}$. Quantum mechanics protocols are directed and intentioned: So is vortical indivension impacting events vortically 'horizontally' -across, and 'vertically' downwards. We proposed that the changes

${ }^{1}$ Communication can go from one dimensional domain to another. We proposed that by applying TDVP essence distinctions, vortical indivension influences 'upstream' results though a specific directed meaningful-(targeted) consciousness - thought. Other facets might also impact including mass-energy components or even targeted infinite gimmel flow. The probability wave collapse or superposition of the quantum receptor is relational and relative to the framework of the observer and consciousness from multiple co-existing states may occur because VI influences dimensionally. Specific meaningful consciousness might produce the effects observed in 3S-1t.

With great respect, beginning in 2011, Neppe and Close appear to have solved some of these problems [15], any of these solutions were in the First Edition of their book, Reality Begins with Consciousness: A Paradigm Shift that Works [15]. But at that point in time (2011 and 2012) [16], the pieces fitted logically together like incomplete jigsaw puzzle pieces, as Neppe and Close were applying their newly enumerated Philosophy of Science principles of Lower Dimensional Feasibility Absent Falsification (LFAF) [106,107,112]. However, the math was not yet demonstrated. We now have been able to prove, mathematically, that many missing pieces of the puzzle can be, and have been, solved [47].

The first problem that most scientists ignored was construing the three dimensions of space and one quantum in time (3S-1t) [48] of our physical experience as a complete paradigm and trying to analyze everything in that context [48]. Yet, there are well over fifty errors or unsolved conundrums in the Standard Model of Physics [52,53,66,113].

These are generally solved, or markedly clarified through the 9-D TDVP model. Possibly the most overt extra component was applied by Nobel Laureate Murray GellMann, who described "gluons" [114,115]. However, this is another example of a math impossibility in 9-D physics and Dimensional Biopsychophysics that tried to explain everything from the Standard Model of Physics. These 'gluons' fit within $3 \mathrm{~S}-1 \mathrm{t}$, implying some extra volume and 'gluing' together of the protons and neutrons components so they don't fly away $[114,115]$. The problem is, applying the geometry of multidimensional volume, gluons are asymmetrical and unstable $[43,57]$, despite the fact that they were (and are) proposed to act just like a 'glue' holding together the nucleus of the atom. They have no other purpose. They cannot be explained in 9-dimensions and mathematically, gluons are impossible [51]. They contradict Fermat's Last Theorem (FLT). This is because there are no electrons making up a third component and a

Table 1: Applying gluons and gimmel to volumetric calculations on the atom of life elements $[52,53]$

\begin{tabular}{|c|c|c|c|}
\hline Substance & Cube & Cube root & Integer? \\
\hline Gluons & $68,697 \mathrm{y}^{3}$ & $40.995338 \mathrm{y}$ & No \\
\hline Gimmel & $125,971,200 \mathrm{y}^{3}$ & $108 \mathrm{y}$ & Yes! \\
\hline
\end{tabular}

Table 2: Tabulated elementary particles including gimmel and TRUE scores

\begin{tabular}{|c|c|c|c|c|c|}
\hline $\begin{array}{c}\text { Elementary } \\
\text { Particle }\end{array}$ & Particle & Mass/energy & $\begin{array}{c}\text { T } \\
\text { Gimmel }\end{array}$ & $\begin{array}{c}\text { Total TRUE } \\
\text { Units }\end{array}$ & $\begin{array}{c}\text { Combined } \\
\text { Particle }\end{array}$ \\
\hline e & electron & 1 & 105 & 106 & Electron = 106 \\
\hline u1 & proton & 4 & 2 & 6 & \\
\hline u2 & proton & 4 & 4 & 8 & \\
\hline d1 & proton & 9 & 1 & 10 & Proton = 24 \\
\hline u3 & neutron & 4 & 5 & 9 & \\
\hline d2 & neutron & 9 & 3 & 12 & \\
\hline d3 & neutron & 9 & 8 & 17 & Neutron = 38 \\
\hline
\end{tabular}


volume (cube) cannot be solved because of FLT [116-119]. There is no balancing third stabilizing component to produce a stable spinning (cubic) combination (Table 1).

In Reality Begins with Consciousness: A Paradigm Shift That Works Edition 1 [43,57], we first hypothesized that reality had to be multi-dimensional beyond the 3 dimensions of space, or the first 4 dimensions (including linear time) in the standard physics model. Subsequently, we were able to demonstrate what we had posited, namely that mathematically, finite reality consists of a 9-dimensional, quantized, volumetric reality [48]. In other words, everything in reality is 3-dimensional. Recognition of this quantized, volumetric fact is very relevant.

However, all of this would still would not work [50] unless there was that third process, gimmel, besides mass and energy $[44,50,51,65,120,121]$. When gimmel is combined with mass and energy in the analysis we have developed, with a basic unit called the Triadic Rotational Unit of Equivalence (TRUE) $[44,57,58,122]$ everything balances perfectly, mathematically.

TRUE applications combine normalized figures for the electrons, and proton and neutron components of the only stable quarks the up and down - quarks with that necessary extra component, gimmel, which is different for each element and compound (Table 2) [43,44,58,59].

Gimmel, as the massless, energyless third component, or substance, and likely the vehicle of consciousness or consciousness itself, is necessary for stability of each and every atom in our universe.

Without gimmel, calculations of stability and symmetry in rotating particles would not work out [60]. The basis of our existing universe is stability and if something becomes unstable and 'decays', for example in microseconds, we don't have any kind of stable universe [124-126]. This is why we can apply the gimmel concepts universally at every level-not only at the level of quantum physics [42], but at the level of our macro reality where, as indicated, the life elements and certain inert ones (neon and helium) (Table 5) have exactly the same and predictably more gimmel than the other elements [52]. The proof of the pudding is in the eating: We hypothesized that the elements that make up life, would have more gimmel than the other elements and they do [43,58]. This appears to be part of nature's amazing hierarchy.

In Table 4, we list the data for many of the lower atomic

Table 3: Tabulation of neptron ${ }^{2}$ subatomic particles gimmel, TRUE and MREV scores for the life elements. $[43,44,58,59]$.

\begin{tabular}{|c|c|c|c|c|} 
Particle & Mass/Energy & $\begin{array}{c}\text { Total TRUE } \\
\text { Gimmel }\end{array}$ & $\begin{array}{c}\text { MREV (minimal rotational } \\
\text { equivalent volumes) }\end{array}$ \\
\hline Electrons $(\mathrm{e})$ & 1 & 105 & 106 & $11,91,016$ \\
\hline Protons $\left(\mathrm{P}^{+}\right)$ & 17 & 7 & 24 & 13,824 \\
\hline Neutrons $\left(\mathrm{N}^{0}\right)$ & 22 & 6 & 38 & 54,872 \\
\hline Totals & 40 & 128 & 168 & $(108)^{3}$ \\
\hline
\end{tabular}

${ }^{2}$ Neptrons: Composite term for Neutrons, Electrons and Protons, as components of the atom. The term introduced by Neppe and Close in 2105.
Table 4: Some Elements And Compounds Including Gap In TRUE Unit Analysis showing Gimmel scores [127].

\begin{tabular}{|c|c|c|c|c|c|}
\hline Compound & $\begin{array}{c}\text { Units } \\
\text { URUE }\end{array}$ & $\begin{array}{c}\text { Total } \\
\text { TR Units }\end{array}$ & $\begin{array}{c}\text { TRUE } \\
\text { Volume }\end{array}$ & $\begin{array}{c}\text { Comments and } \\
\text { Abundance rank\# } 3,4 \\
\text { [128] }\end{array}$ \\
\hline Hydrogen & 150 & 168 & $89.3 \%$ & $(1 \times 108)^{3}$ & Critical Element \\
\hline Deuterium & 128 & 168 & $76 \%$ & $108^{3}$ & Isotope; rare \\
\hline Helium & 256 & 336 & $76.2 \%$ & $(2 \times 108)^{3}$ & Inert Elem ${ }^{6,7} \# 2$ \\
\hline Lithium & 384 & 526 & $73.0 \%$ & $(327.2 \ldots)^{3}$ & Asymmetric \#44 \\
\hline Beryllium & 528 & 710 & $74.4 \%$ & $(437.89 \ldots)^{3}$ & Asymmetric \#44 \\
\hline Boron & 656 & 878 & $74.7 \%$ & $(545.64 \ldots)^{3}$ & Asymmetric \#61 \\
\hline Carbon & 768 & 1008 & $76.2 \%$ & $(6 \times 108)^{3}$ & Organic elem \#4 \\
\hline Nitrogen & 896 & 1176 & $76.2 \%$ & $(7 \times 108)^{3}$ & Life element \#7 \\
\hline Oxygen & 1024 & 1344 & $76.2 \%$ & $(8 \times 108)^{3}$ & Life element \#3 \\
\hline Fluorine & 1,168 & 1,550 & $75.4 \%$ & $(977.22)^{3}$ & Asymmetric \#23 \\
\hline Neon & 1280 & 1680 & $76.2 \%$ & $(10 \times 108)^{3}$ & Inert element \#5 \\
\hline $\mathrm{H}_{2}$ O Water & 1,324 & 1,680 & $78.8 \%$ & $(10 \times 108)^{3}$ & Water \\
\hline Sodium & 1,424 & 1,886 & $75.5 \%$ & $(1,193.12)^{3}$ & Asymmetric \#13 \\
\hline Magnesium & 1536 & 2016 & $76.2 \%$ & $(12 \times 108)^{3}$ & Life element \#9 \\
\hline Aluminium & 1,680 & 2,222 & $75.6 \%$ & $(1,409.06)^{3}$ & Asymmetric \#12 \\
\hline Silicon & 1792 & 2352 & $76.2 \%$ & $(14 \times 108)^{3}$ & Life elem? \#8 \\
\hline Phosphorus & 1,936 & 2,558 & $75.7 \%$ & $(1625.008)^{3}$ & Asymmetric \#16 \\
\hline Sulfur & 2,048 & 2,688 & $76.2 \%$ & $(16 \times 108)^{3}$ & Life elem \#10 \\
\hline Chlorine & 2,192 & 2,894 & $75.6 \%$ & $(1840.97)^{3}$ & Asymmetric \#23 \\
\hline Potassium & 2448 & 3,230 & $75,8 \%$ & $(2056.944 \ldots)^{3}$ & Asymmetric \# 22 \\
\hline Calcium & 2560 & 3360 & $76.2 \%$ & $(20 \times 108)^{3}$ & Life elem \#12 \\
\hline Iron & 3,392 & 4,520 & $75.0 \%$ & $(6096.39)^{3}$ & Asymmetric \#6 \\
\hline & & & & & \\
\hline
\end{tabular}

Table 5: Broader cosmological "Dark" Data (combining dark matter with dark energy) and proportionate gimmel comparisons based on cosmological abundance of elements. Hypothesized valid if within $2 \%$ of observed value.

Volumetric $\Sigma$ (Dark Matter [26.8\%] + Dark Energy [68.3\%]) ratio to cosmology $95.1 \%$ cubed $=86.01 \%$ (Planck probe 2014 data). [64], Gimmel to TRUE ratio (already volumetric) of Abundant Elements $\Sigma$ (volumetric) [Hydrogen $89.3 \%$ * gimmel/TRUE * 0.756 abundance $=67.5 \%]+[$ Helium + less abundant life elements with the same gimmel score $=76.2 \% * 24.4=18.59 \%]=86.09 \%$.

Results: The results not only confirm hypothesis but markedly so with $p<0.001$ difference. The difference between proportions of Dark Matter and Dark Energy together to the ratios of cosmological gimmel $=0.08 \%$. This difference is miniscule 1 in 1,250

number elements. There are the life elements (C, H, O, S, N plus Ca and Mg and likely Silicon) and two inert noble elements ( $\mathrm{He}$ and $\mathrm{Ne}$ ) where applying the Gimmel scores to Triadic Rotational Units of Equivalence (TRUE) calculations, these are all cubic multiples of 108 cubed in score.

Hydrogen is far the most abundant element in the cosmos but describes a unique property: It is likely that great amounts of gimmel are mainly reflected in Hydrogen's absence of a neutron.

We list the first 20 elements in Table 4, plus iron, which is not a life element but apparently contains the most gimmel because of its size. We also show that there are 'GAPS' in this analysis. This data is not only just mathematical, but empirical

${ }^{3} \mathrm{Abundance}$ rank statistics vary markedly depending on whether the cosmos or earth are measured. Therefore two figures existed. However, there is now a third applying the Wolfram statistics and we've used that one.

${ }^{4}$ The 'compensation' for an absent neutron in Hydrogen reflects a critical property and may be a different kind of gimmel which we have technically called 'daled'.

${ }^{5}$ Hydrogen 1 is unique at : $150 / 168=89.2 \%$ gimmel to TRUE ratio. Volumetrically $108^{3}$ $=1,259,712$. 'Daled' vertically 7 produces much more gimmel: 38 for daled instead of a neutron (0 MEUs).

${ }^{6}$ Elem is an abbreviation for element.

${ }^{7}$ Gimmel: 105 for 1 electron (1 mass/energy unit MEU), 7 for 1 proton (17 MEUs), and neutrons are 16 for gimmel; 22 MEUs. 
Table 6: Summary of atomic ratios of dark matter (DM) related to gimmel in nucleons and dark energy (DE) linked with gimmel. [127].

- Research Hypothesis: $<(5 \%-10 \%)$ given the Planck data proportions variation of DE and DM.

- Volumetric (Dark Matter $\left.\left[26.8 \%^{3}\right]=19.25 \%\right)$ / (Dark Energy $\left[68.3 \%^{3}=31.86 \%\right]$ ).

o Consequently this 'dark matter/ dark energy ratio $=60.42 \%$

- Gimmel to TRUE ratio (already volumetric) of (volumetric proportions) of Abundant Elements, $(\Sigma$ [Hydrogen abundance $=70.57 \%]+[$ Helium + less abundant life elements $=29.43 \%$ ]) in (nucleons [protons, neutrons, daled] $=62.10 \%) /($ electron gimmel $=99.06 \%$ )

o Consequently this 'gimmel/TRUE' ratio $=62.69 \%$.

- Results: The difference between the proportions of (Dark Matter to Dark Energy) to the ratios of (nucleon gimmel [linked with quarks and daled] to electron gimmel) is remarkably close: $60.42 \%$ to $62.69 \%$. The results not only confirm the research hypothesis but markedly so with only a $2.27 \%$ difference, far closer than even the reasonable research hypothesis limit.

- Proposals: Dark matter and dark energy must be 'contained' in every stable atom. This can be explained only by applying a multidimensional model, like 9 dimensional spin, not our experiential reality of length, breadth, height in a moment in time ('3S- $1 t^{\prime}$ ).

based on the equivalence with the Mass-energy equivalence normalized data in the CERN Large Hadron Collider ${ }^{8,9}$ $[42,60,61]$.

TRUE analysis also fits with models of dark matter and dark energy [65,129] and is, ostensibly, a necessity for physical stability (Table 5). Asymmetric elements contain less gimmel and are not 108 cubed multiples. Every life element is a cubic multiple of 108 .

These results with dark substances ostensibly link exactly as expected with atomic structure to the extent that dark matter and energy can even be fitted into a 9-dimensional model $[65,129]$ (Table 6).

Together, therefore, we have a unifying model for reality and a single hypothesis that allows one law for all of nature: $[3,130,131]$. There is no longer any quantum weirdness, there are equations in the elements that are logical, and testable in TRUE format, and there are cosmological correlations [132].

Moreover, in order to complete this model, there has to be a necessary infinite continuity [48,131-133], indicating an infinite reality that pervades the finite $[136,137]$. Therefore, there are specifically nine, quantized, finite, volumetric dimensions embedded within the infinite continuity [48,133135]. Gimmel is a key part of this model and is hypothesized to be the major missing part, either consciousness or the vehicle/carrier of consciousness.

These factors, with volumetric phenomena [138], allow for a model that fits, and a metaparadigm that also

${ }^{8}$ Some gap compounds are still undiscovered at both the lower (e.g. 4) and the higher 108 cubed levels e.g. 15, 17, 18 and 19). Some radicals or compounds like water fill the gaps (yellow highlight). When chemicals, compounds or radical exist they still have the properties of $(\mathrm{Nx} 108)$ cubed. The first on that list is helium hydride a super-acid that is not natural $(\mathrm{He} 2 \mathrm{H})$ at $(3 \times 108)^{3} ; \mathrm{HO}$ or $\mathrm{OH} ; \mathrm{H} 2 \mathrm{~N}$; or $\mathrm{CH} 3$ are Gap Radicals as Building Blocks of Amino Acids. Gap at $(9 \times 108)^{3} ;$ Ammonium ion at $\mathrm{NH} 4$ at $(9 \times 108)^{3}$ and at $(13 \times 108)^{3}$ is cysteine radical $s$ an amino acid components. There are some gaps that are not yet explained and although these are all multiples of $(\mathrm{N} \times 108)$ cubed, they do not contain exactly the same proportions of gimmel units: Usually these still are $76.2 \%$ but they may be slightly more up to $80.9 \%$ (ammonium radical $\mathrm{NH} 4$ ) Water is the most important and only complete compound in this analysis and though being a cubic multiple in TRUE volume $(10 \times 108)^{3}$ has $78.8 \%$ not the usual $76.2 \%$.

${ }^{9}$ The 'compensation' for an absent neutron in Hydrogen reflects a critical property and may be a different kind of gimmel which we have technically called 'daled'. actually explains special previously unexplained models such as non-physical life [139-141] and 'ordropy'. Ordropy refers to multidimensional Conservation of Consciousness [142] through the infinite continuity because there is a 'conservation of gimmel' throughout. TDVP therefore creates a comprehensive model of reality as it recognizes that nothing is lost even though in physics there is the entropic physical tendency to disorder of mass and energy [47,143]. Gimmel appears to be the versatile component of the infinite continuity and of the finite dimensions.

But is this purely mathematical? Could it be merelyan operation and not something that is logical at an empirical level? No, because we have shown that our data in TRUE units corresponds exactly with the normalized data from the Large Hadron Collider $[61,91,144]$. Therefore, the TDVP- Triadic Dimensional Vortical Paradigm $[3,145]$, data is both mathematically and empirically based $[61,91,144]$. It is proven [17].

\section{The fundamental components of the triadic dimensional vortical paradigm}

Let's examine the several fundamental components of TDVP.

- First, the concept of dimensions by definition involves measures of extent $[71,146,146]$. Mathematically, there turn out to be 9 specific dimensions, which prior to the proof, we had hypothesized [148]. Extent reflects the measure, such as space and time in physics, and space, time, and gimmel-consciousness in Dimensional Biopsychophysics.

- We need something to measure and fundamental to our physical universe are mass and energy. This allows the idea of 'content'. Content reflects substance, such as mass and energy the empirical measurements that are fundamental to our physical universe. We can express content mathematically relative to extent only indirectly, for example, as famously in Einstein's e = $m c^{2}$ [149]. In this famous equation, matter and energy are shown to be inherently equivalent, and therefore mass can be converted to energy, and their ratio is proportional to the speed of light squared, relating both to measurements of space and time.

- Impact and influence give a control and mechanism to mass and energy allowing extent and content to be impacted, e.g. as in earthquakes.

- We now add a major component namely consciousness into these three-consciousness extent, consciousness content, and consciousness impact and suddenly we have dimensional comparisons of consciousness essence as part of the Calculus of Distinctions [37]. This may be the most important advance of all, particularly after our discovery of gimmel, the third massless, energyless component of subatomic process [44,51,52]. We've been referring here to 'gimmel-consciousness' as the most likely, and almost only explanation. 
- Consciousness has many different ways of being conceptualized $[15,37,39,43]$. Our specific application of Consciousness in this context, constitutes the 'unification of information, knowledge and wisdom at the infinite continuity level'. This infinite consciousness could be expressed in the finite quanta as the equivalent targeted, directed, quantized components of 'meaning'. We humans utilize that meaning as the endpoint expression of our idiosyncratic awareness's in our brain. We do not, therefore, just apply the term 'information' as a synonym for 'Consciousness'. We're conceptualizing something broader than information

- Next in the Triadic Dimensional Vortical Paradigm is a fundamental math and empirical principle namely, volume: Dimensions of content are never points.

o There are no singularities in quantum reality. Singularities are purely conceptual: In reality, these are not points, but volumes.

o One can project a line as linear-as one-dimensional or in one direction, such as the way we conceive of time viz. past-present-future [150,151].

o We can graph in 2 dimensions, as in planes; and on a spreadsheet, but these are artificial measures and even Space must have thickness.

o The most fundamental measure in the laws of nature is volume. Everything is volumetric, and therefore linear dimensions must be cubed. This allows for mathematical calculations that are empirical.

- The principle that follows is everything is quantized. The quantum reflects a limit of minimal quantity. This means that infinitesimal calculus [44,51,52], while valuable of itself, is theoretical, and we've therefore had to develop a new calculus: the calculus of distinctions $[37,39]$.

- It is important to note the mathematical impossibility of our current Standard Model of Physics [66,114]. For example, straight elemental data of protons plus neutrons plus electrons $\neq$ an atom $[48,52,67]$. When you apply these calculations volumetrically, it simply does not work mathematically: it is an inequality [67]. Therefore, there has to be an extra component for such cubic combinations to work mathematically [152]. This introduces integral variables those Diophantine Equations [56,57] and their volumetric solutions necessitate gimmel applying a subset, namely Close's 'Conveyance Equation' $[44,55]$.

- A mathematical area which is likely relevant to add to the TDVP concept of Infinite continuity is Gödel's Incompleteness Theorem (GIT). When analyzing the exact limits of GIT we can recognize that no consistent logical theory can be complete within itself [49]. However, this is very difficult terrain as no matter how many self-consistent logical sentences we record, there will always be one more potentially $\mathrm{N}+1$ more statements out there. Thus, to be 'consistent' one has to go 'outside the box'. That means that GIT here would necessarily require something that is entirely different and outside the consistent logic of the discrete, quantized, finite, volumetric, 3S-1t reality alone. Applying this to extending TDVP to the infinite, we realized that GIT might be fundamental to the TDVP concept of 'Infinite Continuity' as it would not be refuted. In TDVP, we had to create a model that could be applied from outside the standard model, to make it complete, otherwise TDVP could not be an internally consistent Theory of Everything (TOE) $[3,153]$. The hypothesis of over-arching Infinite continuity allows not only for an approach from 'outside the box' but it is also fundamentally different not quantized, but continuous [143].

- The GIT might demand alternative existence of the infinite continuity, to be consistent with its logical axioms. This way there must be a consistence of a logic/set theory that contains the finite quantized in the continuous hypothetical assumption. Our further work in the area suggests of infinite continuity suggests strongly that it is likely to be correct. However, even if infinite continuity did not exist, the rest of TDVP with the 9D and gimmel still would be applicable. But like all other models that apply just the finite reality, it would not be a complete TOE.

- Infinite continuity is a necessary assumption which cannot be directly shown, but is required for any Theory of Everything as otherwise the math model would be necessarily always incomplete [4]. It also is a convenient and feasible hypothesis allowing for further disciplines to traverse such as 'ordropy' [15,141,154,155], enduring multidimensional infinite order (in addition to the entropy of physics with the ultimate tendency towards disorder, despite us living with a lot of order in our 3S-1t sentient existence [156-158], conservation of gimmel in the infinite continuity $[3,135,159,160]$ explanations of infinite existence [139], and meaningful evolution [106,161,162]. Incorporating gimmel also into the infinite continuity component of the TDVP 9-D model provides a single explanation, leading to the Laws of Nature being unified and a consequent philosophical model of Unified Monism [130,131], being proposed, based on the science.

- Fundamental to the Triadic Dimensional Vortical Paradigm are the Triadic Rotational Units of Equivalence (TRUE) $[59,60,123]$. These are measures 
of volumetric equivalents including mass, energy and gimmel. TRUE can be applied to analyzing quantum phenomena, to life elements and other compounds in our macro-world, to dark matter and energy, [61,144] and through the inclusion of gimmel even applied to the infinite continuity [3,153] TRUE analyses, inter alia, show the Triadic Dimensional Vortical Paradigm to be mathematically real [43] and also empirically so. Most definitively, when examining normalized data from the gimmel TRUE unit Mass-energy equivalence scores (GTUs) and comparing these with the CERN Large Hadron Collider, they both are exactly equal integrally with the normalized electron score as 1 , and the proton as 1836 and the neutron as 1839. This proves this component of our Triadic Dimensional Vortical Paradigm data is correct empirically $[42,61]$.

- Next, we have to use the mathematics that are cubic, volumetric, and quantal [43]. That means applying the 'Calculus of Dimensional Distinctions' (CoDD) [37] developed by Ed Close with an assist from Vernon Neppe [37]. The CoDD ensures geometric symmetries which can be applied to the Periodic Table of the Elements. It can be applied to protons, neutrons, and electrons in the atom in all the elements (except Hydrogen). We discover that the elements of life $(\mathrm{C}, \mathrm{H}$, $\mathrm{O}, \mathrm{S}, \mathrm{N}, \mathrm{Ca}, \mathrm{Mg}$, and likely $\mathrm{Si}$; and also $\mathrm{He}$, and $\mathrm{Ne}$ as inert elements) are all multiples of $108^{3}$ TRUE [43], and these are also necessarily stable and symmetrical around an orthogonal axis [43]. These 'life elements' are in union with more gimmel than any other elements [43]. Moreover, Water, ostensibly the most life-sustaining chemical in the universe, has more gimmel than any other compounds [43].

- Finally, and of life-sustaining relevance, is Hydrogen ${ }_{1} \mathrm{H}^{1}$. Hydrogen (specifically ${ }_{1} \mathrm{H}^{1}$ or Protium) is the most abundant element cosmologically. It is the lightest, and it's unique because of the absence of the neutron. This makes ${ }_{1} \mathrm{H}^{1}$ a critically important exception in nature. We have postulated that the Hydrogen ${ }_{1} \mathrm{H}^{1}$ atom contains an extra quantity of 'gimmel' instead of its missing neutron. This results in a far greater quantity of 'gimmel equivalent units' than any other element. However, we cannot prove that this unit that would be an equivalent volumetric replacement for the absent neutron in Hydrogen is 'gimmel' itself. Therefore, we call this neutron-linked extra massless, energyless substance 'daled' [43]. Nevertheless, we strongly propose that this 'daled' replacing the absent neutron, is just another form of gimmel because our calculations applying it appear to have demonstrated this to be so $[37,43]$. This would be the absent-neutron equivalent 'gimmel' that is in union with, for example, the proton of Hydrogen (which contains two up-quarks and one down-quark).
Dr. David Stewart PhD, DNM is a Mathematician, Geophysicist, Earth Scientist, Theologian, Doctor of Natural Medicine and Author of over 300 articles and 17 books. He has a very keen mind and is a critical thinker and might be more familiar with the Close-Neppe work than anyone else in the world. Therefore, he is well-qualified to express an opinion on TDVP, and wrote these words publicly in a nomination letter. This clarifies his opinion for 4D physicists. We had some trepidation including this quotation, but many of our readers and referees have encouraged its inclusion as it would provide valuable insight: This is because most 4D physicists are unfamiliar with 9D+ and gimmel and TDVP. We greatly appreciate Prof. Stewart's kind thoughts, but we do not necessarily agree!

"In summary, I rank Dr. Edward R. Close and Dr. Vernon M. Neppe as peers of the major authors of modern physics and mathematics. I equate them with greats, such as Planck, Einstein, Heisenberg, Schrödinger, Bohr, Dirac, Born, Pauli, Bell, De Broglie, and their predecessors such as Newton, Maxwell, Leibnitz, Kelvin, and many others.

The Neppe-Close work, which is built upon the works of these extraordinarily brilliant and innovating pioneers, has clarified, and extended the science and mathematics that these geniuses originated over a century ago.

The work of Close and Neppe has laid a foundation for all future science to develop. The world of scientific understanding, in all fields, has been permanently changed, and set in a new direction, by the work of Close and Neppe. The future of all mankind is forever brighter because of what they have done. And they aren't finished, yet.

... Dr. Neppe's contributions in both the Medical and Dimensional Biopsychophysics spheres are truly amazing.

I still foresee the day when they will both be awarded other honors, such as a Nobel Prize in Physics. If there were an equivalent award in Mathematics, I would nominate them for that prize, as well."

\section{References}

1. Halpern P. The great beyond: higher dimensions, parallel universes and the extraordinary search for a theory of everything. Hoboken, $\mathrm{NJ}$ : John Wiley \& Sons.2005.

2. Hawking S. The theory of everything. Beverly Hills: New Millennium Press. 2007.

3. Neppe VM, Close ER. A proposed Theory of Everything that works: How the Neppe-Close Triadic Dimensional Distinction Vortical Paradigm (TDVP) model provides a metaparadigm by applying nine-dimensional finite spin space, time and consciousness substrates and the transfinite embedded in the infinite producing a unified reality. IQNexus J. 2014; 16: 1-54.

4. Pokharna SS, Bhandari N, Prajna SC. Jainism and the Theory of Everything (TOE). Transactions of International School for Jain Studies II 2 2. 2018; 1-11. 
5. Kumar M. Quantum: Einstein, Bohr and the great debate about the nature of reality. Thriplow, Cambridge: Icon Books Ltd. 2009.

6. Einstein A. Last Update Date. Relativity and the problem of space. Series Relativity and the problem of space Retrieved Date, Access 1952 (English translation 1954) http://www.relativitybook.com/resources/ Einstein_space.html.

7. Einstein A. Relativity, the special and the general theory-a clear explanation that anyone can understand. London: Routledge and Kegan Paul. 2001.

8. Minkowski H, Lorentz HA, Einstein A, Hermann W, Arnold S, et al. The principle of relativity: a collection of original memoirs: Dover. 1952

9. Einstein A. Relativity, the special and the general theory 15 Edition. New York: Crown Publishers. 1952

10. Aspect A, Grangier P, Roger G. Experimental realization of EinsteinPodolsky-Rosen-Bohm Gedanken experiment: a new violation of Bell's inequalities. Physical Review Letters. 1982; 49: 91-94.1

11. Beichler JE. The five-dimensional continuum approach to a unified field theory. J Paraphysics: 1999; 2: 101-203.

12. Nordström G. On the energy of the gravitational field in Einstein's Theory. Verhandl. Koninkl. Ned. Akad. Wetenschap., Afdel. Natuurk Amsterdam 26. 1918; 1201-1208.

13. Zee A. Quantum field theory in a nutshell. Princeton: Princeton University Press. 2003.

14. Close ER, Neppe VM. The Cabibbo mixing angle and other particle physics paradoxes solved by applying the TDVP multidimensional spin model. IQNexus J. 2014; 1: 13-50.

15. Neppe VM, Close ER. Reality begins with consciousness: a paradigm shift that works (5th Edition) Fifth Edition. Seattle: Brainvoyage.com. 2014.

16. Neppe VM, Close ER. Reality begins with consciousness: a paradigm shift that works (First Edition) 1 Edition. Seattle: Brainvoyage.com. 2012.

17. Neppe VM, Close ER. Applying consciousness, infinity and dimensionality creating a paradigm shift: introducing the triadic dimensional distinction vortical paradigm (TDVP). Neuroquantology. 2011; 9: 375-392.

18. Whitehead AN. Process and reality. New York: Free Press.1978.

19. Griffin DR. Parapsychology and philosophy: A Whiteheadian postmodern perspective. Journal of the American Society for Psychical Research. 1993; 87: 217-288.

20. Griffin DR. Reenchantment without supernaturalism: A Process philosophy of religion Ithaca, NY Cornell University Press. 2001

21. Whitehead AN. Science and the modern world. New York: Free Press 1953.

22. Whitehead AN. Russell B: Principia Mathematica: volumes 1 to 3 . London: Cambridge University Press. 1910.

23. Cantor $\mathrm{G}$ (ed.). Contributions to the founding of the theory of transfinite numbers. New York, Dover, 1955

24. Brown GS. Laws of form. London: Allen and Unwin. 1969.

25. Chalmers DJ. The conscious mind: In search of a fundamental theory. New York: Oxford University Press. 1996.

26. Chalmers DJ. Facing up to the problems of consciousness. J Consciousness Studies, University of Arizona. 1995; 2: 200-219.
27. Shimony A. On mentality, quantum mechanics and the actualization of potentialities, in The large, the small and the human mind. Edited by Penrose R. Cambridge, U.K. Cambridge University Press. 1997; 144-160.

28. Tegmark M. Our mathematical universe: My quest for the ultimate nature of reality. New York: Knopf. 2014.

29. Ferguson AS. Plato's smile of light. (Part II.) The allegory of the cave (Continued). JSTOR. The Classical Quarterly 16: 1. 1922; 636164; 15-28.

30. Editors. Platonism in the philosophy of mathematics, in Stanford encyclopedia of philosophy. Stanford, California. 2013. http://plato. stanford.edu/entries/platonism-mathematics/; http://plato.stanford. edu/entries/platonism-mathematics/

31. Smythies JR. The walls of Plato's cave. Aldershot: Avebury Press.1994.

32. Close ER, Neppe VM. The role of mathematics in investigating the nature of reality (Part 4). IQNexus J. 2015; 7: 22-26.

33. Close ER, Neppe VM. Mathematical and theoretical physics feasibility demonstration of the finite nine dimensional vortical model in fermions. Dynamic International Journal of Exceptional Creative Achievement. 2013; 1301: 1-55.

34. Close ER, Neppe VM. Understanding the calculus of distinctions and its role in TDVP: chapter 8 IQ Nexus J. 2016; 8: 107-114.

35. Close ER, Neppe VM. Further implications: quantized reality and applying Close's Calculus of Distinctions versus the Calculus of Newton(Part 19). IQNexus J. 2015; 7: 110-111.

36. Close ER, Neppe VM. Close's Calculus of Distinctions and Indications. Dynamic International Journal of Exceptional Creative Achievement In press.

37. Close ER, Neppe VM. The Calculus of Distinctions: A workable mathematicologic model across dimensions and consciousness. Dynamic International Journal of Exceptional Creative Achievement. 1213; 1210: 2387 -2397.

38. Baron ME. The origins of the Infinitesimal Calculus. North Chelmsford, MA: Courier Corporation. 1969

39. Lamb H. An elementary course in infinitesimal calculus. Cambridge UK. Cambridge University Press. 1924

40. Close ER, Neppe VM. The proton: Section 6: In: Derivation and application of TRUE quantum calculus for the analysis of quantized reality. 2020

41. Close ER. The calculus of dimensional distinctions, in Elements of mathematical theory of intellect (monograph). Edited by Brandin V Moscow: Moscow Interphysica Lab. 2003; 13-20.

42. Close ER, Neppe VM. Derivation and application of TRUE quantum calculus for the analysis of quantized reality, including empirically verifiable new approaches to mass, neutrons, protons, law of conservation of gimmel and TRUE, TDVP and Deuterium. 2020.

43. Close ER, Neppe VM: Putting consciousness into the equations of science: the third form of reality (gimmel) and the "TRUE" units (Triadic Rotational Units of Equivalence) of quantum measurement. IQNexus J. 2015; 7: 7-119.

44. Close ER, Neppe VM. The TRUE unit: triadic rotational units of equivalence (TRUE) and the third form of reality: gimmel; applying the conveyance equation (Part 12). IQNexus J. 2015; 7: 55-65.

45. Dauben JW: Georg Cantor. His mathematics and philosophy of the ilnfinite. Princeton, NJ: Princeton Uiversity press. 1979.

46. Dauben JW. Georg Cantor and the origins of Transfinite Set Theory. Scientific American. 1983; 248: 122-131. 
47. Neppe VM, Close ER. The Science of reality: Is reality even definable? How the scientific model of the Triadic Dimensional Vortical Paradigm (TDVP) allows for the finite and the infinite. IQ Nexus J. 2019; 11: 4-44.

48. Neppe VM, Close ER. Does the Triadic Dimensional Vortical Paradigm (TDVP) alter the landscape from 4D science to 9D science? The controversy of conventional scientific materialism versus integrating multidimensionality, the infinite and consciousness. IQ Nexus J. 2018; 10: 7-46.

49. Minkowski H. Raum und Zeit. Physikalische Zeitschrift. 1908; 10: 104111.

50. Neppe VM, Close ER. The gimmel pairing: Consciousness and energy and life (Part 13D). IQNexus J. 2015; 7: 122-126.

51. Close ER, Neppe VM. Speculations on the "God matrix": The third form of reality (gimmel) and the refutation of materialism and on gluons. World Institute for Scientific Exploration (WISE) Journal. 2015; 4: 3-30.

52. Neppe VM, Close ER. The Elusive Mystery Solved: The Gimmel of Consciousness: Section 2. In Higher consciousness outside the brain Science and spirituality through the prism of 9-dimensional physics, gimmel and the infinite. IQ Nexus J. 2019; 11: 19-43.

53. Neppe VM, Close ER. Higher consciousness outside the brain: Science and spirituality through the prism of 9-dimensional physics, gimme and the infinite. IQ Nexus J. 2019; 11: 5-43.

54. Close ER, Neppe VM. Elementary particles and units of measurement: applying the conveyance equation (Part 11). IQNexus J. 2015; 7: 48-54

55. Neppe VM, Close ER. Wondrous gimmel: Section 8. In Integrating spirituality into science: applying the Neppe-Close Triadic Dimensional Vortical Paradigm (TDVP). IQNexus J. 2018; 10: 42-47.

56. Neppe VM. Understanding Gimmel with Vernon Neppe. (YouTube) New Thinking Allowed. 2018; https://www.youtube.com/ watch?v=RhV96ShslU4

57. Close ER, Neppe VM. Introductory summary perspective on TRUE and gimmel (Part 1) in Putting consciousness into the equations of science: the third form of reality (gimmel) and the "TRUE" units (Triadic Rotational Units of Equivalence) of quantum measurement IQNexus J. 2015; 7: 8-15.

58. Close ER, Neppe VM. The thirteenth conundrum: introducing an important new concept, TRUE units-Triadic Rotational Units of Equivalence. IQNexus J. 2015; 7: 60-71.

59. Close ER, Neppe VM. Why TRUE units have to be correct: the mass in the proton: re-affirming the truth of Triadic Rotational Units of Equivalence. Chapter 6 IQNexus J. 2016; 8: 122; 70-96.

60. Close ER, Neppe VM. The origin of mass: Section 5: In: Derivation and application of TRUE quantum calculus for the analysis of quantized reality. 2019

61. Close ER, Neppe VM. Application of TRUE analysis to the elements of the periodic table: Section 9: In: Derivation and application of TRUE quantum calculus for the analysis of quantized reality. 2020.

62. Krasznahorkay AJ, Csatlos M, Csige L, Gulyas J, Koszta M, et al. New evidence supporting the existence of the hypothetic X17 particle. 2019. https://arxiv.org/abs/1910.10459

63. Prior R. A 'no-brainer Nobel Prize': Hungarian scientists may have found a fifth force of nature. 2019. https://amp.cnn.com/ cnn/2019/11/22/world/fifth-force-of-nature-scn-trnd/index.html? twitter_impression=true

64. Neppe VM, Close ER. The fourteenth conundrum: Applying the proportions of Gimmel to Triadic Rotational Units of Equivalence compared to the proportions of dark matter plus dark energy: Speculations in cosmology. IQNexus J. 2015; 7: 72-73.

65. Neppe VM, Close ER: Moving to the 9-D reality and gimmel: Part 2. In Does the Triadic Dimensional Vortical Paradigm (TDVP) alter the landscape from 4D science to 9D science? . IQ Nexus J. 2018; 10: 16-20.

66. Neppe VM, with Close ER. The need to refute: Why the Triadic Dimensional Vortical Paradigm (TDVP) entails far more than the Standard Model of Physics: 4D experience is far less than our 9D+ existence. IQNexus J. 2018; 4: 47-78.

67. Neppe VM, Close ER: 4D science: Blindness or logic? Part 1. In Does the Triadic Dimensional Vortical Paradigm (TDVP) alter the landscape from 4D science to 9D science? . IQ Nexus J. 2018; 10: 9-15.

68. Neppe VM, Close ER: The misguided, the cynics, the deniers, the scoffers and the innocent. Part 3. In: Does the Triadic Dimensional Vortical Paradigm (TDVP) alter the landscape from 4D science to 9D science? IQ Nexus J. 2018; 10: 21-27.

69. Neppe VM, Close ER. Speculation versus Proof: Part 4. In: Does the Triadic Dimensional Vortical Paradigm (TDVP) alter the landscape from 4D science to 9D science? IQ Nexus J. 2018; 10: 28-40.

70. Close ER, Neppe VM. Fifteen mysteries of 9 dimensions: on Triadic Rotational Units of Equivalence and new directions, Part III. Neuroquantology. 2015; 13: 439-447.

71. Neppe VM, Close ER. The Cabibbo mixing angle (CMA) derivation: Is our mathematical derivation of the Cabibbo spin mixing angle (CSMA) equivalent? IQNexus J. 2015; 7: 120-128.

72. Figueroa-O'Farrill JM. String theory in a nutshell. 2003. http://www. strings.ph.qmw.ac.uk/Whatls/Nutshell.html

73. Kiritsis E. String theory in a nutshell. Princeton: Princeton University Press. 2007

74. Schwarz P. The official string theory website: Basics. 2003. http:// www.superstringtheory.com/basics/index.html and http://www. superstringtheory.com/forum

75. Brax P. The supermoduli space of Matrix String Theory. 2011. http:// cdsweb.cern.ch/record/411941/files/9912103.pdf

76. Zimmerman Jones A. Hawking and Hertog: String Theory can explain dark energy. 2006, http://physics.about.com/b/2006/06/20/hawking hertog-string-theory-can-explain-dark-energy.htm

77. Close ER, Neppe VM: The fifth conundrum: applying mathematical dimensional extrapolation non-specifically in our dimensional calculations to demonstrate multidimensionality. IQNexus J. 2015; 7 34-38.

78. Close ER, Neppe VM. The sixth conundrum: theoretical knowledge on deriving the Cabibbo angle. IQNexus J. 2015; 7: 39-40.

79. Close ER, Neppe VM: The seventh conundrum: the mathematical derivation of the Cabibbo mixing angle in fermions. IQNexus J. 2015 7: 41-43.

80. Van der Schaar JP. Kaluza Klein theory. http://www-th.phys.rug $\mathrm{nl}$ schaar/htmlreport/node12.html. 1998.

81. Straumann N. Last Update Date. On Pauli's invention of Non-Abelian Kaluza-Klein Theory in 1953. Series On Pauli's invention of NonAbelian Kaluza-Klein Theory in 1953. 2008. http://arxiv.org/pdf/grqc/0012054v1.pdf; http://arxiv.org/pdf/gr-qc/0012054v1.pdf

82. Bailint D, Love A. Last Update Date. Kaluza-Klein theories. Series Kaluza-Klein theories Retrieved Date, Access 1987, http://www.het brown.edu/people/danieldf/literary/eric-KKtheories.pdf; http://www. het.brown.edu/people/danieldf/literary/eric-KKtheories.pdf 
83. Wuensch D. Kaluza-Klein Theory Compendium of Quantum Physics: 2009; 1 : 328-333.

84. Straumann NO. On Pauli's invention of non-abelian Kaluza-Klein Theory in 1953. arXiv:gr-qc/0012054, 2000

85. Rauscher EA, Targ R. The speed of thought: Investigation of a complex space-time metric to describe psychic phenomena. Journal of Scientific Exploration. 2001; 15: 3; 331-354. http://www.scientificexploration. org/jse/abstracts/v15n3a2.html

86. Massimi M. Pauli's Exclusion Principle. Cambridge, England: Cambridge University Press. 2005.

87. Pauli W. Looking for neutrinos, nature's ghost particles (Pauli) SmithsonianMag. 2010. (quotation0.https://www.smithsonianmag com/science-nature/looking-for-neutrinos-natures-ghost-particles64200742/\#Wj8FCmzX0R2PscGm.99

88. Pauli W. Wissenshaftlicher Briefwechsel (Science change), Dialectica: Springer Verlag.1999; 4.

89. Pauli W. Naturwissenschaftliche und erkenntnistheoretische Aspekte der Ideen vom Unbewussten. Dialectica. 1954; 8: 283-301.

90. Close ER, Neppe VM. Derivation and application of TRUE quantum calculus for the analysis of quantized reality, including empirically verifiable new approaches to mass, neutrons, protons, law of conservation of gimmel and TRUE, TDVP and Deuterium. 2019

91. Penrose R. Twistor Algebra. J Mathematical Phy. 1967; 8: 345-366.

92. Penrose SR, Hameroff S. Consciousness in the universe: quantum physics, evolution, brain \& mind. Cambridge: Cosmology Science Publishers. 2011.

93. Hameroff SR. Physical reality and consciousness -- introduction. 2011 http://cognet.mit.edu/posters/Tucson3/Hameroff.Reality.html

94. Penrose R, Rindler W. Spinors and space-time. Cambridge, UK Cambridge University Press. 1986.

95. Penrose R, MacCallum MAH. Twistor theory: An approach to the quantisation of fields and space-time. Physics Report. 1973; 6: 241-315.

96. Penrose R. Non-linear gravitons and curved twistor theory. Gen Rel Grav. 1976; 7: 31-52.

97. Penrose R. Shadows of the mind. London: Oxford University Press. 1994.

98. Penrose R. The road to reality: a complete guide to the laws of the universe. San Francisco: National Books.2007.

99. Hawking S, Penrose R. The nature of space and time. Princeton: Princeton University Press. 1996.

100. Stricker SA. Holographic thermalization in [Formula: see text] super Yang-Mills theory at finite coupling. Eur Phys J C Part Fields. 74: 2727. PubMed: https://www.ncbi.nlm.nih.gov/pubmed/25814879

101. Kostov I, Petkova VB, Serban D. Determinant Formula for the Octagon Form Factor in $\mathrm{N}=4$ Supersymmetric Yang-Mills Theory. Phys Rev Lett 122: $23 ; 231601$

PubMed: https://www.ncbi.nlm.nih.gov/pubmed/31298890

102. Berkovits N. Infinite tension limit of the pure spinor superstring. J High Energy Phy. 2014; 3: 17.

103. Close ER, Neppe VM. One response to "An evaluation of TDVP" with explanations of fundamental concepts. IQ Nexus J. 2019; 10: 79-107.

104. Neppe VM. LFAF: Lower Dimensional Feasibility, Absent Falsification linked with meaningful evolution: Part 5. In Meaningful Evolution, abiogenesis and life solved through gimmel: Translating from the infinite continuity to the discrete finite by applying the Neppe-Close Triadic Dimensional Vortical Paradigm (TDVP). IQNexus J. 2019; 11: 39-43.
105. Neppe VM. Three fundamental models of evolution: Meaningful Evolution (ME), Darwinian Evolution (DE), Intelligent Design (ID). Part 2. In Meaningful Evolution, abiogenesis and life solved through gimmel: Translating from the infinite continuity to the discrete finite by applying the Neppe-Close Triadic Dimensional Vortical Paradigm (TDVP). IQNexus J. 2019; 11: 16-19.

106. Neppe VM. Feasibility and falsification in science: On LFAF (YouTube). New Thinking Allowed. 2018. https://youtu.be/w3elui7unrA

107. Neppe VM, Close ER. Interpreting science through feasibility and replicability: Extending the scientific method by applying "Lower Dimensional Feasibility, Absent Falsification" (LFAF). World Institute for Scientific Exploration (WISE) Journal. 2015; 4: 3-37.

108. Neppe VM, Close ER. Why lower dimensional feasibility (LFAF) Application to metadimensionality Dynamic International Journal of Exceptional Creative Achievement. 2012; 1209: 2352-2360.

109. Neppe VMC, Edward R. Indivension: A way to process the higher dimensional interface. Dynamic International Journal of Exceptional Creative Achievement. 2012; 1209: 2361-2369.

110. Neppe VM, Close ER. Vortical Indivension: A perspective (Part 12) IQNexus J. 2015; 7: 98-111.

111. Neppe VM, Close ER. Quantum probability wave collapse or superposition is explained by vortical indivension (Part 12C). IQNexus J. $2015 ; 7: 3 ; 113$

112. Neppe VM, Close ER. The second conundrum: Falsifiability is insufficient; we need to apply feasibility as well Lower Dimensional Feasibility, Absent Falsification (LFAF) as a scientific method. IQNexus J. 2015; 7: 21-23.

113. Oerter R. The theory of almost everything: the standard model, the unsung triumph of modern physics. New York: Person Education. 2006.

114. Söding P. On the discovery of the gluon. Euro Phy J. 2010; 35: 3-28.

115. Yagi K, Hatsuda T, Miake Y. Quark-Gluon Plasma: From Big Bang to Little Bang: Cambridge monographs on particle physics, nuclear physics and cosmology. (Book 23). Cambridge, England: Cambridge University Press. 2008.

116. Singh S. Fermat's Enigma: The epic quest to solve the world's greatest mathematical problem. Notting Hill, UK: Fourth Estate, Ltd. 1997.

117. Kleiner I. From Fermat to Wiles: Fermat's last theorem becomes a theorem. Elem Math. 2000; 55: 19-37.

118. Close ER. Appendix B: 1965 Proof of Fermat's Last Theorem, in The Book of Atma. Edited by. New York: Libra Publishers. 1977; 93-99.

119. Aczel A/ Fermat's last theorem: unlocking the secret of an ancient mathematical problem. New York: Four Walls Eight WIndows. 1996.

120. Neppe VM, Close ER. Speculations about gimmel Part 5. World Institute for Scientific Exploration (WISE) Journal. 2015; 4: 21-26.

121. Close ER, Neppe VM. More questions answered on the elements, TRUE and gimmel (Part 17). IQNexus J. 2015; 7: 82-102.

122. Close ER, Neppe VM. Understanding TRUE units and gimmel as part of TDVP: chapter 7 IQ Nexus J. 2016; 8: 97-106.

123. Close ER, Neppe VM. The Triadic Dimensional Vortical Paradigm (TDVP) is valid and appropriate: The roles of neutrons and protons, particle emergence including decay and vortical spin - A response. Telicom. 2018; 30: 95-105.

124. Anonymous. Beta decay. 2018; https://en.wikipedia.org/wiki/Beta_ decay. 
125. Basdevant JL, Rich J, Spiro M. $\beta$ decay of nuclei in Fundamentals in nuclear physics: From nuclear structure to cosmology. Edited by Basdevant JL, Rich J, Spiro M. New York. Springer. Chapter 4. 4.3.2, 202. 2005.

126. Neppe VM, Close ER. A nutshell key perspective on the Neppe-Close "Triadic Dimensional Distinction Vortical Paradigm" (TDVP). IQNexus J. 2016; 8: 7-80.

127. Neppe VM, Close ER. A data analysis preliminarily validates the new hypothesis that the atom 'contains' dark matter and dark energy: Dark matter correlates with gimmel in the atomic nucleus and dark energy with gimmel in electrons. IQ Nexus J. 2016; 8: 80-96.

128. Neppe VM, Close ER. Unified monism: linking science with spirituality in a philosophical model. Section 9: In Integrating spirituality into science: applying the Neppe-Close Triadic Dimensional Vortical Paradigm (TDVP). IQNexus J. 2018; 10: 48-51, 2018.

129. Neppe VM, Close ER. The fifteenth conundrum: Applying the philosophical model of Unified Monism: Returning to general principles. IQNexus J. 2015; 7: 74-78.

130. Feynman RP (ed.). The Feynman lectures on physics. USA, AddisonWesley, 1965

131. Neppe VM, Close ER. Relative non-locality and the infinite, in Reality begins with consciousness: a paradigm shift that works (5th Edition). Edited by. Seattle, WA. 2014; 376-379. Brainvoyage.com

132. Neppe VM, Close ER. The discrete finite contained in the continuous infinite: some speculations (Part 13C). IQNexus J. 2015; 7: 120-122.

133. Neppe VM, Close ER. The Triadic Dimensional Distinction Vortical Paradigm (TDVP): The nine-dimensional finite spin metaparadigm embedded in the infinite Dynamic International Journal of Exceptional Creative Achievement. 2014; 1401: 4001-4041.

134. Neppe VM, Pokharna SS, Close ER. The newest of the 10 psi protocols: Introducing Quantum Remote Viewing Clairvoyance: Chapter 8. In: The remarkable Besant-Leadbeater studies in Quantal Clairvoyance (quantal remote viewing) correlate profoundly with the Triadic Rotational Units of Equivalence quantal models in Triadic Dimensional Vortical Paradigm. IQ Nexus J. 2019; 11: 52-56.

135. Neppe VM, Close ER. On Non-locality II: Quantum physics and nonlocality. J Consciousness Exploration and Res. 2015; 6: 97-102.

136. Close ER, Neppe VM. Filling in the gaps of volumetric stability (Part 18). IQNexus J. 2015; 7: 103-109.

137. Neppe V, Close ER. Beyond Physical Life How 'TDVP' (Triadic Dimensional Distinction Vortical Paradigm) explains survival after bodily death. J Spiritual and Consciousness Studies. 2017; 40: 42-62.

138. Neppe VM, Close ER. TDVP and life tracks: Speculations that fit the model (Part 13F). IQNexus J. 2015; 7: 129-130.

139. Neppe VM, Close ER. Reality begins with consciousness: Survival and life itself -Is there a how? Proceedings of 2013 Conference Academy for Spiritual and Consciousness Studies. 2013; 1-17.

140. Neppe VM, Close ER. The Infinite: essence, life and ordropy Dynamic International Journal of Exceptional Creative Achievement. 2012; 1204: 2159-2169.

141. Neppe VM. Building reality from infinite continuity-speculations: Part 4 , in The Science of reality: Is reality even definable? How the scientific model of the Triadic Dimensional Vortical Paradigm (TDVP) allows for the finite and the infinite. IQ Nexus J. 2019; 11: 28-37.

142. Close ER, Neppe VM. The problem of determining the mass of the neutron: Section 7: In: Derivation and application of TRUE quantum calculus for the analysis of quantized reality. 2020
143. Neppe VM, Close ER. TDVP: a paradigm shift that works how the Triadic Dimensional Distinction Vortical Paradigm challenges conventional scientific thinking and explains reality. Telicom. 2014; 27: $24-42$.

144. Neppe VM, Close ER. Dimensions and dilemmas (Part 13A). IQNexus J. 2015; 7: 115-117.

145. Close ER, Neppe VM. Understanding TDVP through dimensions: chapter 5. IQ Nexus J. 2016; 8: 61-69.

146. Close ER, Neppe VM. Support for the hypothesis of a 9-dimensional spin finite reality model (Part 7). IQNexus J. 2015; 7: 33-35.

147. Hecht E. How Einstein confirmed $E=m c^{2}$. Am J Phy. 2011; 79: 591-600.

148. Neppe VM, Close ER. Multidimensional time and 3S-3T-3C (Part 13-J). IQNexus J. 2015; 7: 136-138.

149. Neppe VM, Close ER. Time and Free-will: Section 5. In Free-will: Freedom of choice within limits. IQ Nexus J. 2018; 10: 31-45.

150. Neppe VM, with Close ER. The need to refute: Why the Triadic Dimensional Vortical Paradigm (TDVP) entails far more than the Standard Model of Physics: 4D experience is far less thatn our 9D+ existence. IQNexus J. 2018; 4: 47-78.

151. Smullyan R. Gödel's incompleteness theorems. Oxford: Oxford University Press. 1991

152. Morgart E. The theory of everything has nine dimensions: The sparkling diamond and the quanta jewel turn quantum physics and the nine-pronged world of consciousness-on its ear. USA Today Magazine. 2014; 66-68.

153. Neppe VM, Close ER. Reality Begins with Consciousness (RBC) Glimpses and Glossary 2nd Edition. Seattle, WA: Brainvoyage.com. 2014.

154. Neppe VM, Close ER. The relevance of Free-will of the Neppe-Close models as reflected in Reality Begins with Consciousness: Section 4. In Free-will: Freedom of choice within limits. IQ Nexus J. 2018; 10 : 23-31.

155. Houran J. Entropy and environmental mystery: a parapsychological perspective. Percept Mot Skills. 2007; 105: 688-690. PubMed: https://www.ncbi.nlm.nih.gov/pubmed/18065094.

156. Sturrock PA. A Bayesian maximum-entropy approach to hypothesis testing, for application to RNG and similar experiments. Journal of Scientific Exploration. 1997; 11: 181-192.

157. May EC, P. SSJ, James CL. Shannon entropy as an intrinsic target property: Toward a reductionist model of anomolous cognition, in The Parapsychological Association 37th Annual Convention. August 7-10, 1994 University of Amsterdam. Edited by Bierman, Dick J.1994.

158. Neppe VM, Close ER. Special concepts in the finite and infinite anomalous process (Part 13). IQNexus J. 2015; 7: 114-122.

159. Neppe VM, Close ER. The infinite (Part 13B). IQNexus J. 2015; 7: 117-120.

160. Neppe VM. Meaningful Evolution ('ME'): Triadic Dimensional Vortical Paradigm (TDVP) And Consciousness: A Refutation Of Darwinian Evolution ('DE') and a scientific addition or alternative to Intelligent Design ('ID'): Part 3 In Meaningful Evolution, abiogenesis and life solved through gimmel: Translating from the infinite continuity to the discrete finite by applying the Neppe-Close Triadic Dimensional Vortical Paradigm (TDVP). IQNexus J. 2019; 11: 20-25.

161. Neppe VM. Perspective: why 'meaningful evolution' is a formidable model of evolution: Part 8. In Meaningful Evolution, abiogenesis and life solved through gimmel: Translating from the infinite continuity to the discrete finite by applying the Neppe-Close Triadic Dimensional Vortical Paradigm (TDVP). IQNexus J. 2019; 11: 62-76. 